\title{
Investigation of cold Rb Rydberg atoms in a magneto-optical trap
}

\author{
D. B. Tretyakov ${ }^{1}$, I. I. Beterov ${ }^{1}$, V. M. Entin ${ }^{1}$, I. I. Ryabtsev ${ }^{1}$ and P. L. Chapovsky ${ }^{2}$ \\ ${ }^{1}$ Institute of Semiconductor Physics, Prospekt Lavrentyeva 13, 630090 Novosibirsk, Russia \\ ${ }^{2}$ Institute of Automation and Electrometry, Prospekt Koptyuga 1, 630090 Novosibirsk, Russia
}

(Dated: September 4, 2008)

\begin{abstract}
We present our results on the experiments with cold Rb Rydberg atoms in a magneto-optical trap (MOT). Characteristic features of our experiment were the excitation of Rydberg atoms in a small volume within the cold atom cloud and sorting of the measured signals and spectra over the number of registered Rydberg atoms. We have measured the effective lifetime of the Rydberg state $37 P$, as well as its polarizability in a weak electric field. The results are in good agreement with the theoretical calculations. We have shown that localization of the small excitation volume around the zero-magnetic-field point makes possible to increase the spectral resolution and to obtain narrow microwave resonances in Rydberg atoms without switching off the MOT quadrupole magnetic field. We have measured the dependence of the amplitude of the dipole-dipole interaction resonances on the number of Rydberg atoms, which has a linear character and agrees with the theory for weak dipole-dipole interaction.
\end{abstract}

PACS numbers: 32.80.Rm, 32.70.Jz, 03.67.Lx

\section{INTRODUCTION}

In recent years the cold atoms in highly excited Rydberg states have attracted increasing attention due to the fact that the gas consisting of almost frozen Rydberg atoms behaves in a way similar to amorphous solids, in which the strong collective inter-atomic interactions can lead to broadening and shift of the spectral lines [1-3], as well as to ionization of atoms and formation of ultracold plasma [4].

Long-range interactions of cold Rydberg atoms are of particular interest due to their possible application to quantum computing. The basic ideas were formulated in paper [5] for two-qubit logical gates and in [6] for the effect of dipole blockade at the collective excitation of an ensemble of cold atoms. Two-qubit operations can be realized at short excitation of two adjacent Rydberg atoms, whose interaction leads to variation in the phase of the collective wave function. The dipole blockade is an effect of Rydberg excitation of only one atom in the ensemble because the resonances of multiatom excitation are shifted due to the interatomic interactions. Both effects can be realized using the dipole-dipole interaction of Rydberg atoms. However, until now they have not been observed experimentally for a small number of atoms, and thus become a key issue in the possible realization of a quantum computer based on neutral atoms.

Thanks to their large dipole moments, Rydberg atoms can effectively interact at distances of several and even tens of microns. The two pioneering experiments $[7,8]$ report on the influence of van der Waals interaction on the probability and spectra of the optical transitions with principal quantum number $n>60$. The effect is interpreted as "van der Waals blockade" and is analogous to

*Electronic address: ryabtsev@isp.nsc.ru the dipole blockade. In papers [9-12] a resonance microwave radiation was used to control the dipole-dipole interaction.

The dynamics of the signals of the resonant dipoledipole interaction was investigated in paper [13], where the dependences of the Rydberg states population on the interaction time and number of atoms have been studied. Finally, the experimental works $[14,15]$ describe the first attempts to observe the effect of dipole blockade. The resonant interaction caused a decrease in the Rydberg atom excitation probability of several times. However, dipole blockade effect with excitation of a single atom has not yet been reported.

Despite the large number of publications devoted to investigation of long-range interactions of Rydberg atoms, many aspects of these interactions remain unstudied, especially for a small number of atoms. This is because of the fact that Rydberg atoms were mainly registered using micro-channel-plate detectors, which do not provide resolution over the number of atoms. At the same time, in a series of experiments performed by our group [16,17], for registration of $\mathrm{Na}$ Rydberg atoms in a thermal beam we used a channel electron multiplier (channeltron) VEU-6, which allowed registration and sorting of signals according to the number of detected atoms (from 1 to 5 ). In the present work we apply for the first time this approach to cold Rydberg atoms.

Further we present the first results of our experiments with cold Rb Rydberg atoms. A feature of these experiments is the excitation of a few Rydberg atoms in a small volume within the cold atomic cloud in a MOT. The aim of the work was realization of excitation in a small volume, development of diagnostic methods for cold Rydberg atoms, spectroscopy of the microwave transitions between Rydberg states in the presence of the MOT quadrupole magnetic field, as well as observation of dipole-dipole interaction of a small number of Rydberg atoms. 

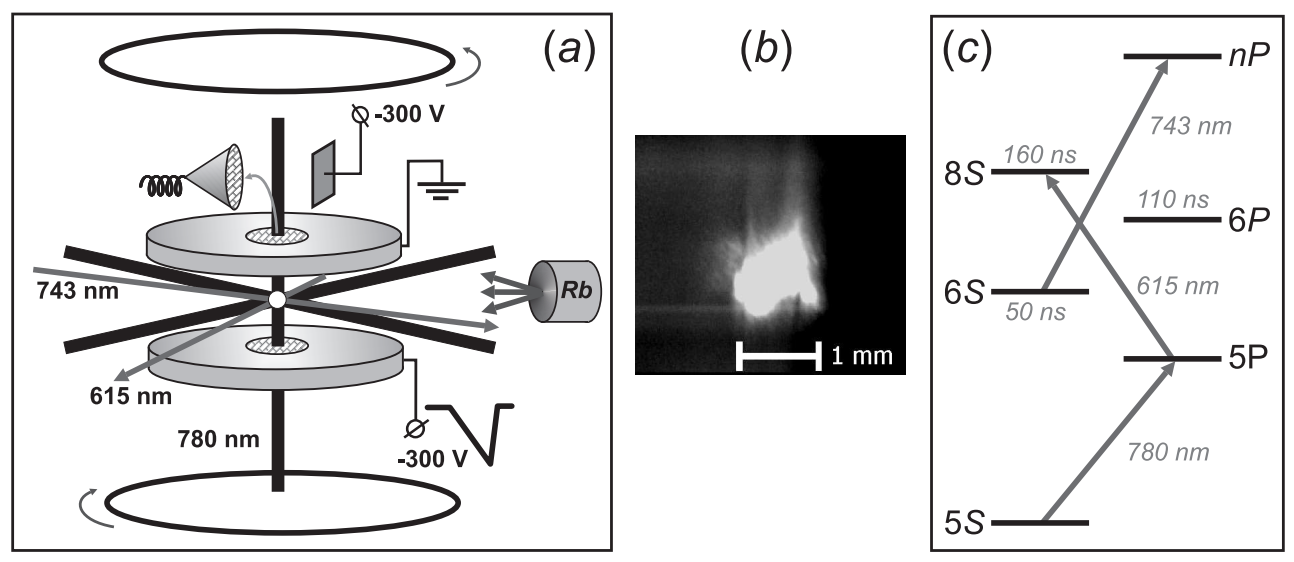

FIG. 1: (a) Schematic diagram of the experiment with cold Rb Rydberg atoms in a MOT. (b) Image of the cold Rb atom cloud after setting up the meshes on the vertical cooling laser beams (the shadows of the meshes with a cell size of $0.7 \mathrm{~mm}$ can be seen). (c) Energy level scheme for the three-step laser excitation of the Rb $n P$ Rydberg states.

\section{EXPERIMENTAL SETUP}

The experiments were performed with cold Rb atoms in a MOT, illustrated in Fig.1(a). The MOT has a standard configuration [18] and consists of a vacuum chamber with optical windows, an oven as a Rb source, and anti-Helmholtz coils providing 3-dimensional magnetic field gradient of $10-15 \mathrm{G} / \mathrm{cm}$ in the MOT center. The laser cooling system is based on two external-cavity diode lasers at the $780 \mathrm{~nm}$ wavelength. The atom cooling was realized by means of three pairs of mutually orthogonal light beams, each pair consisting of counterpropagating light waves of opposite circular polarization. One of the lasers (the cooling laser, with power $50 \mathrm{~mW}$ and linewidth of $1 \mathrm{MHz}$ ) was tuned to the closed $5 S_{1 / 2}(F=3) \rightarrow 5 P_{3 / 2}(F=4)$ transition of the ${ }^{85} \mathrm{Rb}$ isotope, with a red detuning of $20-30 \mathrm{MHz}$. The second laser (repumping laser, with a power of $10 \mathrm{~mW}$ and linewidth of $1 \mathrm{MHz}$ ) was in resonance with the $5 S_{1 / 2}(F=2) \rightarrow 5 P_{3 / 2}(F=3)$ transition.

After adjustment and stabilization of the laser wavelength, a cloud of cold atoms of about $1 \mathrm{~mm}$ diameter was formed in the center of the MOT. The loading time of the MOT depended on the alignment of the laser beams and the $\mathrm{Rb}$ source temperature and was on the order of $1-3 \mathrm{~s}$. The temperature of the trapped atoms was not measured, but it is known from literature that the typical values are $100-300 \mu \mathrm{K}$ [18]. For example, in ref. [19] a similar MOT was reported and its temperature, measured using recoil-induced resonances, was about $140 \mu \mathrm{K}$.

The excitation and registration of Rydberg atoms was performed between the two stainless-steel plates, with a $10 \mathrm{~mm}$ hole in the center of each plate [Fig.1(a)]. In order to create a homogeneous electric field, these holes were closed with optically transparent (85\% transmission) metallic meshes. The distance between the plates was $10 \mathrm{~mm}$. Electric field was used for detection of the Rydberg atoms by means of selective field ioniza- tion (SFI) [20]. The electrons formed after ionization were accelerated by the electric field, flied through the upper mesh, after which a deflecting electrode directed them towards the channeltron's input window. Its output pulses were processed by a fast analog-to-digital converter, gated integrator and a computer. This allowed us to control the number of atoms and the population of Rydberg states for a large range of the principal quantum number $n$.

The transmission of the vertical cooling laser beams through the meshes leads to inhomogeneities of the intensity distribution and distortion of the cloud shape. The cloud image, taken with a CCD-camera, is shown in Fig.1(b). The shadows of the meshes with cell size about $0.7 \mathrm{~mm}$ are clearly seen. Nevertheless, in the center of the cloud the atomic distribution is homogeneous enough, which made possible to vary the position of excitation of the Rydberg atoms. In order to determine the total number of atoms in the cloud, the CCD camera was calibrated with respect to the intensity of the resonant fluorescence. Typically $10^{5}-10^{6}$ atoms were observed, which corresponds to a density of the cold atoms of $10^{8}-10^{9} \mathrm{~cm}^{-3}$.

The excitation of cold $\mathrm{Rb}$ atoms into the Rydberg states $n P(n=30-50)$ was performed in three steps [Fig.1(c)]. For the first step $5 S_{1 / 2} \rightarrow 5 P_{3 / 2}$ the cw cooling laser was used. The second step $5 P_{3 / 2} \rightarrow 8 S_{1 / 2}$ was excited using the radiation of a pulsed Rhodamine $6 \mathrm{G}$ dye-laser with a wavelength of $615 \mathrm{~nm}$, pumped with the second harmonic of Nd:YAG laser at a pulse repetition rate of $5 \mathrm{kHz}$. The $8 S$ state has a lifetime of $160 \mathrm{~ns}$ and decays into the lower P-states, including the $6 P$-level. The latter has a lifetime of $110 \mathrm{~ns}$ and quickly decays into the $6 S$ state having a lifetime of 50 ns. Calculations made in the kinetic-equations approximation have shown that about $10 \%$ of the atoms from the $8 S$ state cascade down to the $6 S$ state. Further, as a third step, the Rydberg $n P$ states were excited from $6 S$ by means of a pulsed Ti:Sapphire laser with a wavelength of $743 \mathrm{~nm}$, 
pumped by the second harmonic of Nd:YAG laser at a pulse repetition rate of $5 \mathrm{kHz}$. Both pulsed lasers were synchronized in time and their pulse width was about $50 \mathrm{~ns}$.

The radiations for the second- and third-step excitation were focused onto the cold atom cloud in crossedbeams geometry (Fig.1) by means of objectives with a focal length of $80 \mathrm{~mm}$. The beam waist diameters measured at a level of $1 / \mathrm{e}^{2}$ were $25 \pm 5 \mu \mathrm{m}$ for the dye-laser and $40 \pm 5 \mu \mathrm{m}$ for the Ti:Sapphire laser. Thus, the intersection of the focused beams formed the effective excitation volume of Rydberg atoms whose dimensions were around $40-100 \mu \mathrm{m}$, depending on the mutual position of the beam waists and on the presence or absence of transition saturation.

Microwave spectroscopy [20] was used for diagnostics of the cold Rb Rydberg atoms in the MOT. The microwave transitions between Rydberg states with $n=30-50$ have natural linewidths on the order of several $\mathrm{kHz}$ and their frequencies are in the range of $30-100 \mathrm{GHz}$. As a source of microwave radiation we used a carcinotron oscillator G4-142, whose frequency with a linewidth of $20 \mathrm{kHz}$ was locked to a quartz synthesizer and was scanned in the interval 53-82 GHz. The radiation was introduced directly through the MOT window. Since the saturation intensity of the single-photon transitions is around $10^{-12}-10^{-9} \mathrm{~W} / \mathrm{cm}^{2}$, they are easily excited with lowintensity radiation and detected with SFI. The microwave transition spectra provide information on the presence of external magnetic and electric fields and their spatial distribution. Such fields can be used for manipulating the energy levels of the Rydberg atoms.

The time diagram of the signals in the detection system is shown in Fig.2(a). Following each laser pulse exciting some of the cold atoms to an initial Rydberg state $n P$, the atoms interacted with each other or with the microwave radiation for a time period $t_{0}=1-10 \mu \mathrm{s}$. After that, a ramp of the ionizing electric field was applied with a rise time of about $2 \mu \mathrm{s}$. Depending on the Rydberg state of the atom, ionization took place at different moments in time after the laser pulse. The pulsed ionization signal was then registered at the output of VEU-6 using two gates, corresponding to the initial $n P$ and final $n^{\prime} L$ state of the Rydberg atom. The number of electrons registered for a single laser pulse was determined by the number of Rydberg atoms in the excitation volume and by the detection efficiency of VEU-6 [17].

Figure 2(b) shows a histogram of the amplitude of the output pulses from VEU-6. Several peaks can be seen, corresponding to the different numbers of registered Rydberg atoms (from 1 to 5 ). The mean number of atoms, detected per laser pulse, was 2.2. After each laser pulse, the data acquisition system measured the amplitude of the output VEU-6 signal at both registration channels (for the initial and final state), and determined the number of atoms according to the histogram, measured in advance. After accumulation of data for 1000-5000 laser pulses, it sorted the signals over the number of atoms

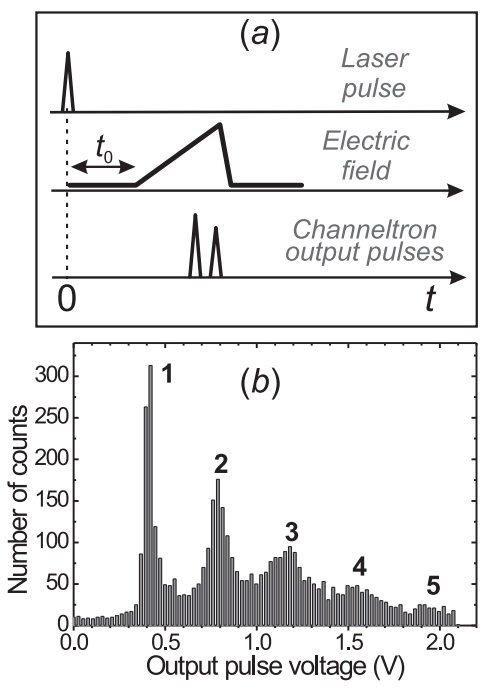

FIG. 2: (a) Time diagram of the pulses in the experiments with selective field ionization (SFI) of Rydberg atoms. (b) Histogram of the amplified output pulses of the channel multiplier VEU-6.

and calculated the probability of the transition from the initial to the final Rydberg state.

\section{LIFETIME MEASUREMENT}

First, a test measurement was performed to ensure that the registered signals are from the cold Rydberg atoms and not from the thermal atomic beam from the $\mathrm{Rb}$ source. For this, the effective lifetime $\tau_{\text {eff }}$ of the Rydberg atoms in the MOT was determined. We have chosen as a test level the initial Rydberg state $37 P$. The lifetime of the $n P$ states with other $n$ values can be estimated with the approximate scaling dependence $\tau_{\text {eff }} \sim n^{3}$.

It should be noted that in atomic-beam experiments the measurement of the lifetime of high Rydberg states is not a trivial task, since the time of flight of the atoms through the detection system is usually much smaller than their lifetime. The situation with the cold Rydberg atoms in a MOT is quite opposite. For a temperature of the $\mathrm{Rb}$ cloud of $200 \mu \mathrm{K}$, the root-mean-square (rms) velocity of the atoms is $25 \mathrm{~cm} / \mathrm{s}$, so at a timescale of milliseconds the cloud does not expand significantly. Thus, direct measurement of the lifetime is possible even for states with $n \sim 100$. The first lifetime measurements of cold $\mathrm{Rb}$ Rydberg atoms are reported in ref. [21]. In a later work [22] it was proposed that the experiments in ref. [21] would yield overestimated values of the lifetimes.

In our experiments we measured the dependence of the mean number of Rydberg atoms in the $37 P$ state on the time delay of the SFI pulse (time $t_{0}$, see Fig.2). Fig.3 shows the experimental records, made for cold atoms in the working MOT (black curve) and for hot atoms when the MOT magnetic field is off (grey curve). 


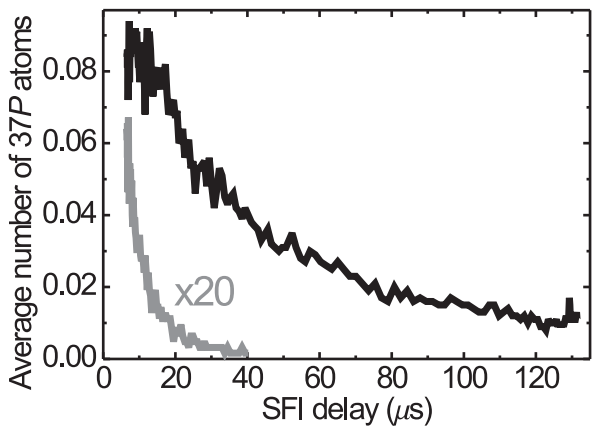

FIG. 3: Dependence of the mean number of atoms in the $37 P$ state on the time delay after the exciting laser pulse: black curve - for cold Rydberg atoms in the working MOT, grey curve - for hot atoms after switching off the MOT magnetic field.

Both dependences are well described with the exponent $\exp \left(-t_{0} / \tau_{\text {eff }}\right)$. The measured lifetime of the cold atoms was $\tau_{\text {eff }}=(38 \pm 3) \mu \mathrm{s}$. This value is close to the previously calculated by us in ref. [23] value of $43 \mu \mathrm{s}$ taking into account the lifetime decrease due to background blackbody radiation at $300 \mathrm{~K}$. However, it noticeably differs from the experimental value of $47 \mu$ s reported in ref. [21], which could support the conclusions drawn in [22].

At the same time, for the hot atoms the effective lifetime was only $\tau_{\text {eff }}=(6 \pm 0.5) \mu$ s. It is determined by the time of flight of the atoms through the detection system. Moreover, from Fig.3 it can be seen that the signal from the hot atoms is about 20 times weaker than the signal from the cold ones. Therefore, we could conclude that in a working MOT the contribution of hot atoms to the measured signal is less than $5 \%$ and can be neglected.

\section{INFLUENCE OF EXTERNAL FIELDS}

Atoms in MOTs are trapped using an inhomogeneous quadrupole magnetic field created by antiHelmholtz coils. Typical values of the field gradient are $10-15 \mathrm{G} / \mathrm{cm}$, thus the variation of the magnetic field within the atomic cloud of $1 \mathrm{~mm}$ in size is around $1 \mathrm{G}$. This field leads to Rydberg levels splitting of the order of 1-3 MHz, which limits the spectral resolution in experiments with cold Rydberg atoms in a MOT. To overcome this, often the magnetic field is turned off prior to measurements $[11,24]$. However, the fall time for the field down to $50-100 \mathrm{mG}$ depends on the inductance of the coils and on the eddy currents in the metallic elements of the detection system and the vacuum chamber. Therefore it is difficult to be made less than several milliseconds, and typical values are $10-20 \mathrm{~ms}$. This leads to a decrease in the signal accumulation rate and to nonsteadiness of the cold atomic cloud due to its gradual expansion. In addition, the slow switching of the mag-
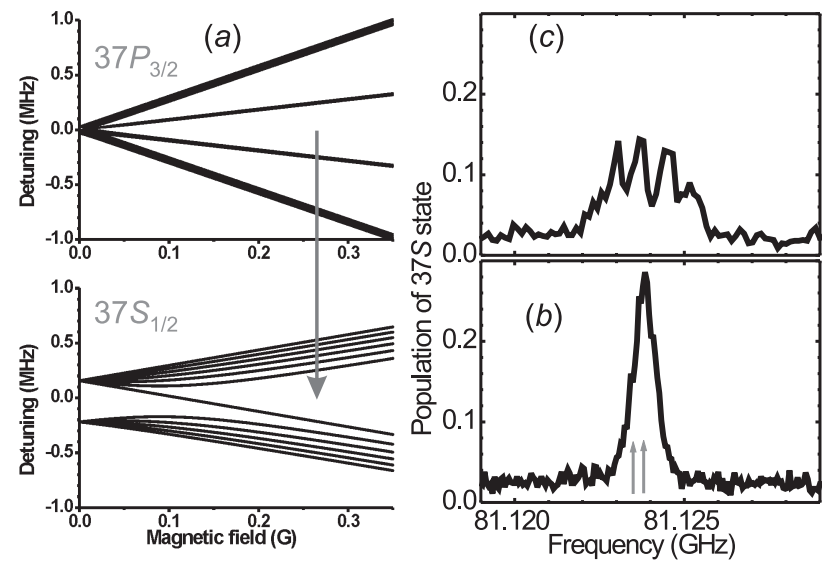

FIG. 4: (a) Energy level scheme for the microwave transition $37 P_{3 / 2} \rightarrow 37 S_{1 / 2}$ in ${ }^{85} \mathrm{Rb}$ atoms in a weak magnetic field, numerically calculated for the nonlinear Zeeman effect on the states $37 P_{3 / 2}$ and $37 S_{1 / 2}$. (b) Spectrum of the microwave transition $37 P_{3 / 2} \rightarrow 37 S_{1 / 2}$ when the excitation volume is localized in the center of the cold atomic cloud and (c) at its periphery. The arrows indicate the calculated positions of the unresolved hyperfine-structure components.

netic field does not comply with the requirements to the quantum computer qubits, which should be manipulated on timescales of less than microsecond [16].

Therefore, in our experiments the magnetic field was not switched off. This provided high signals accumulation rate, determined by the exciting laser pulse rate $(5 \mathrm{kHz})$. In order to improve the spectral resolution and to decrease the influence of the inhomogeneous magnetic field, we localized a small excitation volume of Rydberg atoms close to the center of the cold atomic cloud where the field turns to zero.

The influence of the magnetic field was studied by microwave spectroscopy of the transition $37 P_{3 / 2} \rightarrow 37 S_{1 / 2}$ in ${ }^{85} \mathrm{Rb}$. The $37 S_{1 / 2}$ state has two hyperfine sublevels $F=2,3$ with hyperfine splitting of $376 \mathrm{kHz}$, and the $37 P_{3 / 2}$ state has four hyperfine sublevels $F=1,2,3,4$ with a total width of $62 \mathrm{kHz}$ (these values were calculated from the data for the lower-lying states using the scaling dependence $n^{-3}$ ). However, even a weak magnetic field can cause full mixing and splitting of the hyperfine levels, as can be seen from the calculated by us diagram in Fig.4(a). If the hyperfine structure is not taken into account, the energy shifts of the magnetic sublevels of the fine levels $M_{J}$ are described with the simple formulas $[25]:$

$$
\Delta E=g \mu_{B} B M_{J}, \quad g\left(S_{1 / 2}\right)=2, \quad g\left(P_{3 / 2}\right)=4 / 3,
$$

where $g$ is the Lande factor, $\mu_{B}-$ the Bohr magneton, and $B$ - the magnetic field. The values of $g$ were calculated using the general formula for the fine structure components of the $S$ and $P$ states [25]. 
The spectrum of the microwave transition $37 P_{3 / 2} \rightarrow$ $37 S_{1 / 2}$ was expected to be different for different positions of the excitation volume within the atomic cloud. This is confirmed by Fig.4, which shows the spectra obtained for single-atom signals when the excitation volume of about $100 \mu \mathrm{m}$ was close to the center of the cloud (b) and to its periphery (c). In the center, the profile is almost Lorentzian, with a full width at half maximum (FWHM) of $670 \pm 30 \mathrm{kHz}$. This width is determined mainly by the unresolved hyperfine structure of the $37 S_{1 / 2}$ state and the time of interaction of the atoms with the microwave field $(3 \mu \mathrm{s})$, which sets the Fourier-transform resonance width to about $300 \mathrm{kHz}$. At the same time, at the cloud periphery the profile consists of four components with a total spectrum width of $2.7 \mathrm{MHz}$. The magnetic field magnitude, estimated using Eq.(11), was $0.35 \pm 0.05$ G. Thus, such an experiment on localization of the Rydberg excitation into a small volume demonstrates a sort of "tomography" of the cold atomic cloud. It also confirms the possibility to obtain narrow resonances in an inhomogeneous magnetic field, under the condition that the excitation volume is localized around the zero magnetic field position. The latter is important for the provision of strong dipole-dipole interaction of Rydberg atoms, since even a weak magnetic field leads to decrease in the interaction energy by several times [11].

Rydberg atoms are also very sensitive to external electric fields, since the polarizability of the Rydberg states increases as $n^{7}$. As an illustration, Fig.5 shows the spectrum of the microwave transition $37 P_{3 / 2} \rightarrow 37 S_{1 / 2}$ in a weak electric field. The $S$ and $P$ states of the Rb atoms are subject to quadratic Stark effect [see the scheme in Fig.6(a)]. The $37 P_{3 / 2}$ state is split into two components with momentum projections $\left|M_{J}\right|=1 / 2$ and $\left|M_{J}\right|=3 / 2$. The splitting is determined by the tensor polarizability of the $37 P_{3 / 2}$ level, which has been measured to be $\alpha_{2}\left(37 P_{3 / 2}\right)=-(3.1 \pm 0.15) \mathrm{MHz} /(\mathrm{V} / \mathrm{cm})^{2}$. This value agrees well with our numerical calculations, which give $\alpha_{2}\left(37 P_{3 / 2}\right)=-3.2 \mathrm{MHz} /(\mathrm{V} / \mathrm{cm})^{2}$. At the same time, the level shifts are determined by the difference in scalar polarizability of the $37 P_{3 / 2}$ and $37 S_{1 / 2}$ levels. The experimentally measured value of $\alpha_{0}\left(37 P_{3 / 2}\right)-\alpha_{0}\left(37 S_{1 / 2}\right)=$ $-(30 \pm 1.5) \mathrm{MHz} /(\mathrm{V} / \mathrm{cm})^{2}$ is also close to the calculated value of $-29.5 \mathrm{MHz} /(\mathrm{V} / \mathrm{cm})^{2}$.

It should be noted that the increase in the electric-field strength to $4.66 \mathrm{~V} / \mathrm{cm}$ leads to resonance broadening by about $3 \mathrm{MHz}$. This is due to the electric field inhomogeneity over the excitation volume, which is relatively small - only $(0.5 \pm 0.1) \%$, but still limits the spectral resolution.

\section{RESONANT DIPOLE-DIPOLE INTERACTION}

As can be seen from the results of the previous experiment, it is possible to control the Rydberg state energies using a weak electric field. In particular, it can be used to

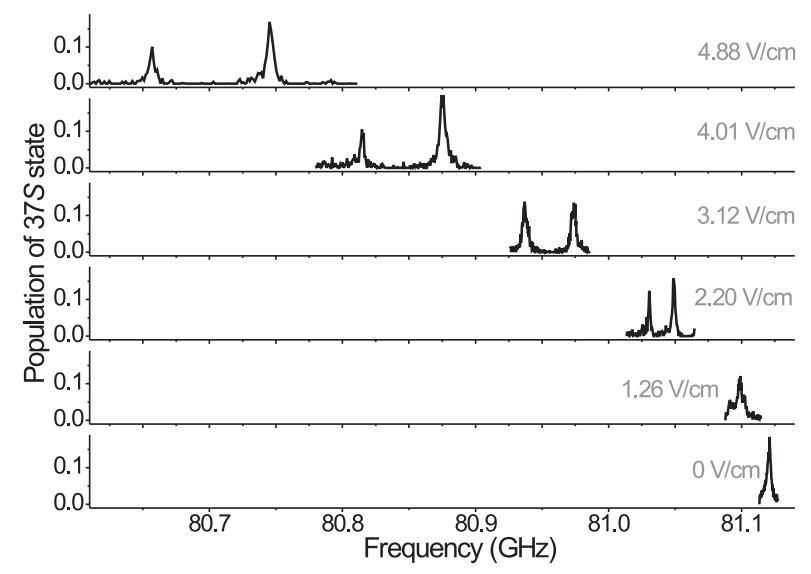

FIG. 5: Spectrum of the microwave transition $37 P_{3 / 2} \rightarrow$ $37 S_{1 / 2}$ in a weak electric field.

Stark-tune the resonant dipole-dipole interaction of the Rydberg atoms [26], which is required for practical realization of quantum-logic operations and dipole blockade $[6,27]$. In the case of $\mathrm{Rb}$ atoms in $n P$ Rydberg state, the resonant interaction of two atoms is realized as $[13,28]$ :

$$
\begin{aligned}
& \operatorname{Rb}\left(n P_{3 / 2}\right)+\operatorname{Rb}\left(n P_{3 / 2}\right) \rightarrow \\
& \operatorname{Rb}\left(n S_{1 / 2}\right)+\operatorname{Rb}\left((n+1) S_{1 / 2}\right)
\end{aligned}
$$

The exact energy resonance for this process can be achieved by means of Stark tuning of the Rydberg levels in an electric field [Fig.6(a)]. It should be noted, though, that because of the specific values of the quantum defects and polarizabilities, resonance Stark tuning is possible only for states with $n \leq 38$. Table 1 provides a list of the calculated by us initial detunings and mean critical values of the electric field for the states $n=35-40$. In our experiment, the chosen initial state was $37 P_{3 / 2}$, since for the higher state $38 P_{3 / 2}$ the critical electric field value for the double resonance is rather small, and thus the resonance can be broadened due to stray fields, while for lower states the frequencies of the microwave resonances are out of the working range of the microwave oscillator

\begin{tabular}{|c|c|c|}
\hline \multicolumn{3}{|c|}{$\begin{array}{l}\text { Table 1. Calculated values of the initial detuning } \\
\Delta_{0} \text { and critical field } E_{c r} \text { for the double Stark reso- } \\
\text { nances from } n P \text { states shown in Fig.6(a) }\end{array}$} \\
\hline$n$ & $\Delta_{0}(\mathrm{MHz})$ & $E_{c r}(\mathrm{~V} / \mathrm{cm})$ \\
\hline 35 & 382 & 4.5 \\
\hline 36 & 228 & 3.1 \\
\hline 37 & 105 & 1.9 \\
\hline 38 & 5.6 & 0.4 \\
\hline 39 & -73 & \\
\hline 40 & -136 & \\
\hline
\end{tabular}
used for Rydberg-state diagnostics. 
At resonance the cross-section of the process (2) strongly increases. As a result of the interaction, one of the atoms goes into the lower state $n S_{1 / 2}$, and the other - into the higher state $(n+1) S_{1 / 2}$. The atomic-state change is registered using the SFI method. As a rule, in the experiments the total population of the $n S_{1 / 2}$ state is measured, since in the $\mathrm{Rb}$ atoms the $(n+1) S_{1 / 2}$ state has almost the same critical field for SFI as the $n P_{3 / 2}$ state. There can be more than two interacting atoms within the excitation volume, if they are closely spaced. In this case collective interactions in a cold Rydberg gas take place [3].

As mentioned earlier, the specific feature of our experiments is the possibility to determine the number of Rydberg atoms $N$ and their states after each laser pulse. The signals from 1000-5000 pulses are accumulated and subsequently automatically sorted over the number of atoms, and the transition probability for each group is calculated. As a result, the following signals are measured:

$$
S_{N}=\frac{n_{N}(37 S)}{n_{N}(37 P)+n_{N}(37 S)+n_{N}(38 S)} .
$$

Here $n_{N}(n L)$ is the total number of detected Rydberg atoms in a state $n L$ for the case of $N$ Rydberg atoms. In fact, the signal $S_{N}$ describes the mean transition probability for each atom after interaction with the $N-1$ surrounding atoms.

The resonant dipole-dipole interaction is registered through the dependence of the signals $S_{N}$ on the strength of the dc electric field. In zero field, the signals have a small background related to the non-resonant transitions induced by blackbody radiation and atomic collisions. When the electric field is tuned to resonance, the signal increases due to the increased cross-section of the process (2). The amplitude and width of the resonances for cold Rydberg atoms depend on the energy and the duration of the dipole-dipole interaction.

The operator of the dipole-dipole interaction between two atoms $a$ and $b$ is written as:

$$
\hat{V}_{a b}=\frac{1}{4 \pi \varepsilon_{0}}\left[\frac{\hat{\mathbf{d}}_{a} \hat{\mathbf{d}}_{b}}{R_{a b}^{3}}-\frac{3\left(\hat{\mathbf{d}}_{a} \mathbf{R}_{a b}\right)\left(\hat{\mathbf{d}}_{b} \mathbf{R}_{a b}\right)}{R_{a b}^{5}}\right] .
$$

Here $\hat{\mathbf{d}}_{a}$ and $\hat{\mathbf{d}}_{b}$ are the dipole moment operators for the atoms $a$ and $b, \mathbf{R}_{a b}$ is a vector connecting the two atoms, and $\varepsilon_{0}-$ the dielectric constant. To calculate the evolution of the Rydberg state population, it is necessary to solve the problem for a quasimolecule formed by the two atoms $[13,17]$. For motionless Rydberg atoms this problem can be solved analytically. The resulting evolution of the population of the final state $37 S$ in each atom is described by the following expression:

$$
\rho_{2}\left(t_{0}\right) \approx \frac{1}{2} \frac{2 \Omega_{a b}^{2}}{2 \Omega_{a b}^{2}+\Delta^{2} / 4} \sin ^{2}\left(\sqrt{2 \Omega_{a b}^{2}+\Delta^{2} / 4} t_{0}\right),
$$

where $\Omega_{a b}=V_{a b} / \hbar$ is the dipole-dipole interaction energy in frequency units, and $\Delta=\left(2 \mathrm{E}_{37 P}-\mathrm{E}_{37 S}-\mathrm{E}_{38 S}\right) / \hbar$ is the detuning from exact resonance. Thus, the interaction between two motionless Rydberg atoms leads to oscillations in the final state population of each atom, in a way similar to the Rabi oscillations in a two-level atom. Since this process is coherent, it can be used for realization of two-qubit logical operations and of quantum logic gates based on neutral atoms.

It is impossible to obtain a general analytical formula for the transition probability $\rho_{N}\left(t_{0}\right)$ for a larger number of interacting atoms. This is due to the fact that besides the resonant dipole-dipole interaction (2), it is necessary to take into account the exchange processes like $\mathrm{Rb}(37 P)+\mathrm{Rb}(37 S) \rightarrow \mathrm{Rb}(37 S)+\mathrm{Rb}(37 P)$ and $\mathrm{Rb}(37 P)+\mathrm{Rb}(38 S) \rightarrow \mathrm{Rb}(38 S)+\mathrm{Rb}(37 P)$, which are always resonant and lead to a population exchange between neighboring atoms [3]. If these processes are not taken into account, then Eq.(5) should be simply modified by substituting $2 \Omega_{a b}^{2}$ with the sum of the squares of the interaction energies for all atomic pairs, and by replacing the scaling factor $1 / 2$ by $1 / N$ :

$$
\begin{aligned}
\rho_{N}\left(t_{0}\right) \approx & \frac{1}{N} \frac{\sum_{a \neq b} \Omega_{a b}^{2}}{\sum_{a \neq b} \Omega_{a b}^{2}+\Delta^{2} / 4} \times \\
& \sin ^{2}\left(\sqrt{\sum_{a \neq b} \Omega_{a b}^{2}+\Delta^{2} / 4} t_{0}\right)
\end{aligned}
$$

Here the total transition probability is calculated as the sum of the probabilities over all possible binary interactions. Such an approximation is valid for a weak dipoledipole interaction, when the $S$-states are weakly populated and $\rho_{N}\left(t_{0}\right)<<1$. The numerical modeling which takes into account the exchange interactions confirms the applicability of Eq.(6) for this case.

The signals $S_{N}$ measured in our experiment, however, cannot be directly modeled with Eq.(6). First of all, this expression is obtained for the two motionless atoms with a fixed separation and fixed dipole orientation. In our experiment the signals are accumulated from Rydberg atoms with arbitrary position within the excitation volume and with arbitrary dipole orientations. Therefore, it is necessary to average Eq.(6) over interatomic distances and dipole orientations. Second, as was shown in our earlier work [17], due to the finite detection probability of the SFI detector, the signals $S_{N}$, corresponding to detection of $N$ Rydberg atoms, are a mixture of signals from a larger number of actually excited atoms $i \geq N$ :

$$
S_{N}=\mathrm{e}^{-\bar{n}(1-T)} \sum_{i=N}^{\infty} \rho_{i}\left(t_{0}\right) \frac{[\bar{n}(1-T)]^{i-N}}{(i-N) !},
$$

where $\bar{n}$ is the mean number of Rydberg atoms excited per laser pulse, and $T$ - the detection efficiency of the SFI detector. 

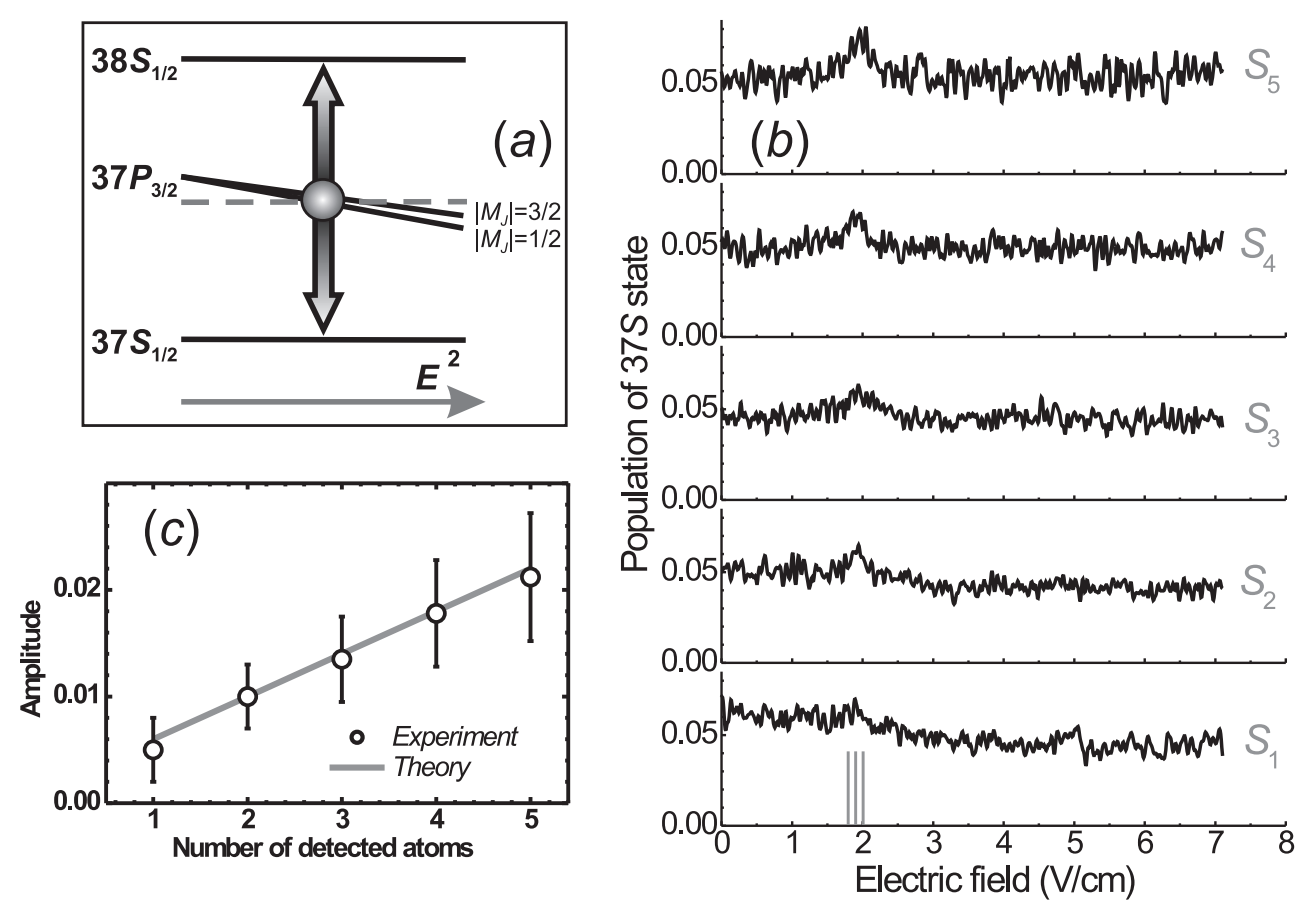

FIG. 6: (a) Scheme of the appearance of the double Stark resonance $37 S_{1 / 2}-37 P_{3 / 2}-38 S_{1 / 2}$ in the electric field $E=$ $1.8-2.0 \mathrm{~V} / \mathrm{cm}$. (b) Spectra of the resonant dipole-dipole interaction for selectively detected 1 to 5 Rydberg atoms. The vertical lines denote the calculated positions of the resonances. (c) Dependence of the resonance amplitude on the number of detected Rydberg atoms. The dots represent experimental data, and the line is theoretical dependence for weak dipole-dipole interaction.

Nevertheless, it is possible to use Eq.(6) for estimation of the resonance amplitude and width, if the mean distance between the Rydberg atoms in the excitation volume is introduced and the dipole orientation is not considered (scalar model). Since the summation in Eq.(6) is over $N(N-1)$ terms, we can write:

$$
\sum_{a \neq b} \Omega_{a b}^{2} \equiv N(N-1) \bar{\Omega}^{2}
$$

where $\bar{\Omega}$ is the rms energy of the dipole-dipole interaction of the two Rydberg atoms with arbitrary positions within the excitation volume. For weak interaction Eq.(6) yields for the resonance amplitude:

$$
\rho_{N}\left(t_{0}, \Delta=0\right) \approx(N-1) \bar{\Omega}^{2} t_{0}^{2},
$$

In this case the resonance width is the same for all $N$ and is determined only by the interaction time $t_{0}$. Substituting (9) into (7) and summing over $i$, we obtain:

$$
S_{N} \approx[N-1+\bar{n}(1-T)] \bar{\Omega}^{2} t_{0}^{2},
$$

Thus, at weak dipole-dipole interaction the $S_{N}$ dependence on $N$ should be linear. At $N=1$ the signal $S_{1}$ is due to the imperfection of the SFI detector, and it characterizes the measurement error in the experimental signals.

In our experiment the observation of dipole-dipole interaction was performed at the maximal concentration of the cold atoms of $10^{9} \mathrm{~cm}^{-3}$. The excitation volume of about $100 \mu \mathrm{m}$ in size was localized close to the center of the cold atomic cloud. The time of free interaction of the Rydberg atoms was $t_{0} \approx 2.2 \mu \mathrm{s}$. The resonant dipole-dipole interaction spectra were obtained for 1 to 5 registered Rydberg atoms. These spectra are presented in Fig.6(b). The vertical lines indicate the calculated positions of the three possible resonances occurring at $1.79,1.89$ and $2.0 \mathrm{~V} / \mathrm{cm}$ for the different Stark components of the $37 P_{3 / 2}$ state. As can be seen from the experimental spectra, the individual resonances overlap and form a single resonance with a width of about $250 \mathrm{mV} / \mathrm{cm}$. Presumably, the resonance broadening was due to the electric field inhomogeneity over the excitation volume $(10-20 \mathrm{mV} / \mathrm{cm})$, as well as due to the stray ac fields whose amplitude was estimated to be up to $30-50 \mathrm{mV} / \mathrm{cm}$.

The broadening of the resonance lead to the low signal amplitude and signal-to-noise ratio, as seen from Fig.6(b). Nevertheless, it was possible to measure the amplitude versus the number of detected Rydberg atoms [Fig.6(c)]. The obtained dependence is linear, which 
agrees well with the theoretical prediction of Eq.(10).

Moreover, using the experimental dependence shown in Fig.6(c) and the method proposed by us in [17], it was possible to determine the unknown detection efficiency of Rydberg atoms $T=(40 \pm 15) \%$ and the mean number of excited Rydberg atoms $\bar{n}=(1.7 \pm 0.5)$. This measurement was based on the measured amplitude ratio of the two- and one-atom resonances $S_{2} / S_{1} \approx 2 \pm 0.7$. The mean number of registered atoms in the present experiment $\bar{n} T=0.7 \pm 0.1$, needed for the above estimations, was experimentally obtained from a histogram similar to that presented in Fig.2(b).

The above experimental values and Eq. (10) make possible to estimate the unknown parameter $\bar{\Omega}^{2} t_{0}^{2}=0.004 \pm$ 0.0015 . Thus, for an interaction time of $2.2 \mu \mathrm{s}$ we can estimate the rms energy of the dipole-dipole interaction of the two atoms in the excitation volume to be $\bar{\Omega} / 2 \pi=4.7 \pm 1.6 \mathrm{kHz}$. This value is close to the theoretical estimation of $3 \mathrm{kHz}$, obtained for a mean distance between the atoms $R=50 \mu \mathrm{m}$ and for radial parts of the dipole moments 1320 and 1290 a.u. of the transitions $37 P_{3 / 2} \rightarrow 37 S_{1 / 2}$ and $37 P_{3 / 2} \rightarrow 38 S_{1 / 2}$, respectively, which were calculated numerically by us.

It should be noted, that the increase in supply voltage of VEU-6 up to $3.5 \mathrm{kV}$ lead to increase in $T$ of three times as compared to work [17], where the supply voltage was $2.5 \mathrm{kV}$. The high detection probability is a necessary prerequisite in the experiments on quantum logic gates, since the measurement error depends on $\bar{n}(1-T)$, as can be seen from Eq.(10).

Further experiments on the investigation of the dipoledipole interaction of a small number of Rydberg atoms in an excitation volume of $20-30 \mu \mathrm{m}$ are of interest. It is expected that the decrease in excitation volume and better shielding against stray electric fields would allow for the observation of narrower resonances due to the decrease in magnetic and electric field inhomogeneity, as well as would increase the resonance amplitude due to the decrease in the mean distance between the atoms.

\section{CONCLUSION}

In this paper we presented the results of our first experiments with cold Rb Rydberg atoms in a MOT. The experiments have revealed the main features of the excitation and detection of Rydberg atoms in a small excitation volume within the cold atom cloud. It has been shown that due to the cooling and trapping of atoms, the effective lifetime of the cold Rydberg atoms in a MOT is close to the natural one. This makes possible to perform measurements at a large timescale, taking all advantages of Rydberg atoms.

The localization in a small excitation volume allows to select the position of atomic interaction within the inhomogeneous external fields, and to map the spatial distribution of these fields using microwave spectroscopy. In particular, localizing the excitation volume close to the zero magnetic-field point improves the spectral resolution and yields narrow microwave resonances even without turning off the MOT quadrupole magnetic field.

The main characteristic feature of the present experiment was the detection of Rydberg atoms by means of a channel electron multiplier. This enables the sorting of the obtained signals over the number of detected atoms for each laser pulse. As a result, it was possible to measure the dependence of the amplitude of the dipole-dipole interaction resonance on the number of Rydberg atoms, which agrees well with the theory for weak dipole-dipole interaction. The obtained results are of interest for the development of quantum logic gates based on cold Rydberg atoms.

\section{Acknowledgments}

The authors are grateful to Christina Andreeva for fruitful discussions and help in preparing this paper. The work was supported by RFBR (grant No. 05-02-16181) and by the Russian Academy of Sciences.
[1] W.R. Anderson, J.R. Veale, and T.F. Gallagher, Phys. Rev. Lett. 80, 249 (1998).

[2] I. Mourachko, D. Comparat, F. de Tomasi, A. Fioretti, P. Nosbaum, V.M. Akulin, and P. Pillet, Phys. Rev. Lett. 80, 253 (1998).

[3] V.M. Akulin, F. de Tomasi, I. Mourachko, and P. Pillet, Physica D 131, 125 (1999).

[4] M.P. Robinson, B. Laburthe Tolra, M.W. Noel, T.F. Gallagher, and P. Pillet, Phys. Rev. Lett. 85, 4466 (2000).

[5] D. Jaksch, J.I. Cirac, P. Zoller, S.L. Rolston, R. Cote, and M.D. Lukin, Phys. Rev. Lett. 85, 2208 (2000).

[6] M.D. Lukin, M. Fleischhauer, R. Cote, L.M. Duan, D. Jaksch, J.I. Cirac, and P. Zoller, Phys. Rev. Lett. 87, 037901 (2001).

[7] D. Tong, S.M. Farooqi, J. Stanojevic, S. Krishnan, Y.P. Zhang, R. Cote, E.E. Eyler, and P.L. Gould, Phys. Rev. Lett. 93, 063001 (2004).

[8] K. Singer, M. Reetz-Lamour, Th. Amthor, L.G. Marcassa, and M. Weidemüller, Phys. Rev. Lett. 93, 163001 (2004). 
[9] I. Mourachko, Wenhui Li, and T.F. Gallagher, Phys. Rev. A 70, 031401(R) (2004).

[10] K. Afrousheh, P. Bohlouli-Zanjani, D. Vagale, A. Mugford, M. Fedorov, and J.D.D. Martin, Phys. Rev. Lett. 93, 233001 (2004).

[11] K. Afrousheh, P. Bohlouli-Zanjani, J.D. Carter, A. Mugford, and J.D. D. Martin, Phys.Rev.A. 73, 063403 (2006).

[12] P. Bohlouli-Zanjani, J.A. Petrus, and J.D.D. Martin, Phys. Rev. Lett. 98, 203005 (2007).

[13] S. Westermann, T. Amthor, A.L. de Oliveira, J. Deiglmayr, M. Reetz-Lamour, and M. Weidemüller, Eur. Phys. J. D 40, 37 (2006).

[14] T. Vogt, M. Viteau, J. Zhao, A. Chotia, D. Comparat, and P. Pillet, Phys. Rev. Lett. 97, 083003 (2006).

[15] T. Vogt, M. Viteau, A. Chotia, J. Zhao, D. Comparat, and P. Pillet, Phys. Rev. Lett. 99, 073002 (2007).

[16] I.I. Ryabtsev, D.B. Tretyakov, and I.I. Beterov, J. Phys. B 38, S421 (2005).

[17] I.I. Ryabtsev, D.B. Tretyakov, I.I. Beterov, and V.M. Entin, Phys. Rev. A 76, 012722 (2007); Erratum: Phys. Rev. A 76, 049902(E) (2007).

[18] H. Metcalf, P. Van Der Straten, and H.E. Stanley, Laser cooling and trapping, Springer, New York (1999).

[19] P.L. Chapovsky, JETP Lett. 86, 78 (2007).
[20] Rydberg States of Atoms and Molecules, edited by R.F. Stebbings and F.B. Dunning, Cambridge University Press, Cambridge (1983); T.F. Gallagher, Rydberg Atoms, Cambridge University Press, Cambridge (1994).

[21] A.L. de Oliveira, M.W. Mancini, V.S. Bagnato, and L.G. Marcassa, Phys. Rev. A 65, 031401(R) (2002); V.A. Nascimento, L.L. Caliri, A.L. de Oliveira, V.S. Bagnato, and L.G. Marcassa, Phys. Rev. A 74, 054501 (2006).

[22] D.A. Tate, Phys. Rev. A 75, 066502 (2007).

[23] I.I. Beterov, D.B. Tretyakov, I.I. Ryabtsev, A. Ekers, N.N. Bezuglov, Phys. Rev. A 75, 052720 (2007).

[24] J. Han, Y. Jamil, D.V.L. Norum, P.J. Tanner, and T.F. Gallagher, Phys. Rev. A 74, 054502 (2006).

[25] I.I. Sobelman, An Introduction to the Theory of Atomic Spectra, Pergamon, Oxford (1972).

[26] K.A. Safinya, J.F. Delpech, F. Gounand, W. Sandner, and T.F. Gallagher, Phys. Rev. Lett. 47, 405 (1981).

[27] M. Saffman and T.G. Walker, Phys. Rev. A 72, 042302 (2005).

[28] A.L. de Oliveira, M.W. Mancini, V.S. Bagnato, and L.G. Marcassa, Phys. Rev. Lett. 90, 143002 (2003). 